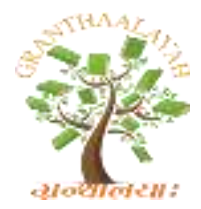
INTERNATIONAL JOURNAL OF RESEARCH -
GRANTHAALAYAH
A knowledge Repository

Science

\title{
NON-LINEAR EFFECTS OF AIRFOIL FORCE DATA ON DESIGN PERFORMANCE OF A LOW-REYNOLDS NUMBER PROPELLER
}

\author{
Umunna J Reuben ${ }^{1}$, Koju Hiraki ${ }^{2}$, Miyamoto Shohei ${ }^{3}$ \\ 1, 2, 3 Space Dynamic Laboratory, Kyushu Institute of Technology, Japan
}

\begin{abstract}
Over the last three decades and a half, there has been huge effort to develop high performance propellers suitable for flight in the rarefied Mars atmosphere. This paper describes work undertaken in validating vortex theory in the design of a 2-bladed heavily loaded propeller with a solidity of $\approx 0.25$ and chord based Reynolds number of $\approx 60 \mathrm{k}$ (calculated at $75 \%$ radius) at design point. The design was based on minimum induced propeller losses and lifting line theory. 2Dairfoil experiment data of SD7037 collected at Reynolds number of 60k was used for the entire blade design. At design advance ratio, more than $50 \%$ of the entire blade radius operated between 40k - 60k Reynolds numbers. A design goal of the propeller was to minimize variation in Reynolds number from hub to tip radius. Wind tunnel tests carried out at Kyushu Institute of Technology were performed in two (2) ways: constant angular velocity and changing airflow velocity over the propeller, and constant airflow velocity and changing propeller angular velocity. The fabricated propeller showed good agreement in efficiency for both test cases. However, considerable discrepancy was observed between theory and experiment in thrust and power. Investigation showed that non-linearity associated with airfoil aerodynamic data not captured by linearization result in a less representative modeling of the airfoil force coefficient and consequently, discrepancy in propeller performance between theory and experiment.
\end{abstract}

Keywords: Propeller Design; Low-Reynolds Number; Airfoil Data; Xrotor.

\section{Nomenclature:}

$\begin{array}{ll}\mathrm{T} & =\text { Thrust } \\ \mathrm{B} & =\text { Number of blades } \\ \rho & =\text { Density } \\ \mathrm{r} & =\text { Station radius } \\ \mathrm{rtip} & =\text { Tip radius } \\ \mathrm{rhub} & =\text { Hub radius } \\ \mathrm{VR} & =\text { Resultant velocity } \\ \phi & =\text { Local flow angle } \\ \mathrm{CD} & =\text { Drag coefficient } \\ \mathrm{CD}(0) & =\text { Minimum CD } \\ \mathrm{CL} & =\text { Lift coefficient }\end{array}$


$\mathrm{CL}(0)=\mathrm{CL}$ at $\mathrm{CD}(0)$

$\operatorname{Re}=$ Reynolds number

c $\quad=$ Blade chord

$\omega=$ Angular velocity

$\mathrm{vt} \quad=$ Induced tangential velocity

$\mathrm{V}=$ Free airstream velocity

va $=$ Induced axial velocity

$\mathrm{J}=$ Advance ratio

$\mathrm{CT}=$ Thrust coefficient

$\mathrm{CD}=$ Drag coefficient

$\mathrm{a} 0=$ Lift curve slope

()$(\mathrm{E})=$ Experiment

()$(\mathrm{J})=$ Advance ratio

()(i) = Induced inflow angles

Cite This Article: Umunna J Reuben, Koju Hiraki, and Miyamoto Shohei (2018). "NON-LINEAR EFFECTS OF AIRFOIL FORCE DATA ON DESIGN PERFORMANCE OF A LOWREYNOLDS NUMBER PROPELLER." International Journal of Research Granthaalayah, 6(8), 196-213. https://doi.org/10.29121/granthaalayah.v6.i8.2018.1450.

\section{Introduction}

Recently there has been considerable interest in Low-Reynolds flights driven by efforts to develop micro air bots for various applications: achieving flights within the stratosphere and in the rarefied Mars atmosphere. For a Mars flight, extremely low fluid inertia and speed of sound limits the propeller tip speeds thus adding to the design complexity. Other design consideration includes; compactness of the design to ensure fit into aerosols from where it would be deployed for operation on entry into Mars atmosphere. Hence, blades with large radius would need to be folded and reliably deployed before the commencement of flight. The propeller design presented in this work was not designed to meet the propulsion demands of any specific air vehicle but rather the goal was to validate the application of lifting line theory and minimum induced losses in the design of high solidity, low Reynolds number operating propellers. The works of Betz [1], Goldstein [2] all assumes light loading for which their respective formulation holds true. Mark Drela replaced the advance ratio $(\mathrm{v} / \Omega \mathrm{R})$ with wake advance ratio to account for heavy disk loading [3]. However, the implementation of graded momentum formulation in Xrotor notes that the momentum formulation is unsuitable for advance ratios greater than 0.5 [4].

Muller [5] described chord based Low Reynolds number as flows less than $10^{\wedge} 6$. However, Reynolds numbers $<70 \mathrm{k}$ are of particular interest in this work. A major challenge that must be overcome towards the realization of low-Reynolds number-high performance flight is an efficient propulsion system. Studies favor a propeller based propulsion system for high efficiency, long endurance flights at high altitude or rarefied Martian atmosphere where propellers may operate at Reynolds numbers lower than 100k. Youngren [6] used Xrotor to redesign a COT propeller and demonstrated that improving the performance of 2D airfoil and optimally distributing propeller load along its radius could enhance the performance of COT propellers operating at Low Reynolds numbers. Wind tunnel tests of the redesigned propeller conducted by Youngren showed better 
performance than Xrotor predictions. Smedresman et al [7] used Qprop, a code based on lifting line theory, to create a propeller that operates at about 21000 chord based Reynolds number, a value that is below the interest of this work. The propeller design utilized 2D aero data of a modified MA409 airfoil that was obtained using Xfoil. Wind tunnel test of the fabricated propeller showed 20\% lower efficiency from Qprop predictions. The uncertainties associated with 2D airfoil force coefficients were reported by Smedresman to be partly responsible for the discrepancy between experiment and predicted results. Dexter et al [8] tested several low Reynolds number COT propellers. Experiment data from their wind tunnel tests showed performance dependency on Reynolds number. However, details of airfoil and related force coefficients used in the respective designs of the COT propellers as expected are not in the public domain. As a result, it was difficult to compare design performance data with experimental data. In the performance tests of Black widow's propeller, Grasmeyer et al [9] achieved excellent performance agreement between experimental data and lifting line theory predictions. However, details of $2 \mathrm{D}$ airfoil aerodynamic data and propeller operational Reynolds number were not supplied.

The design of a high performance propeller requires the accurate knowledge of the 2D airfoil data to be used for the design. Poor or inaccurate 2D airfoil data results in poor propeller performance and discrepancy between vortex theory prediction and experiment data. For most flows over an airfoil at Reynolds number below 100k, Laminar Separation Bubble (LSB) is not only present, but it also degrades the airfoil performance. Selig [10] suggests that LSB progresses forward towards the leading edge with increasing angle of attack until total flow separation of the flow from the trailing edge is achieved due to "burst" of the laminar bubble eventually causing airfoil stall. Presence of LSB modifies the airfoil shape and consequently the airfoil aerodynamic force coefficients. Depending on the geometry, position and radial spread along a propeller, LSB can drastically affect the overall performance of a propeller.

\section{Experiment Set Up}

Two different experiment set-ups were used for the measurement of thrust and torque. Figure 1(a) and Figure 1(b) depicts the set-up for thrust and torque respectively. A TCLZ-NA load cell was used for the measurement of thrust while torque reading was collected via a UM-II torque meter having an accuracy of 0.0001 . In the measurement of thrust and torque, a DC power source supplied power to a Hyperion ZS2213-22 electric motor via an ATLAS 21A ESC. A WF1974 match function generator was used to send PWM command to the electronic speed controller. The angular velocity of propeller was obtained from a HT-5500 contactless tachometer and free airstream velocity was collected using a digital anemometer. Propeller performance measurements were carried out using the Kyushu Institute of Technology wind tunnel. The wind tunnel is an open-ended no-return Eiffel tower type with an open test section. The propeller was mounted about $0.3 \mathrm{~m}$ away from the exit plane of the convergent section of the open test area that measures $0.5 \mathrm{~m}$ x $0.5 \mathrm{~m}$ x $1 \mathrm{~m}$. 


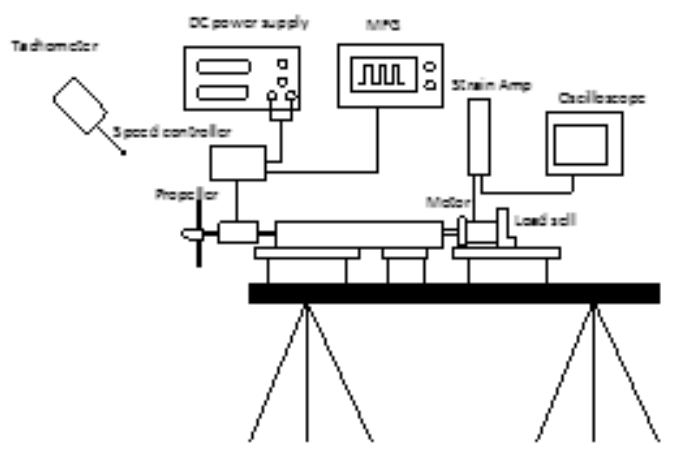

(a)

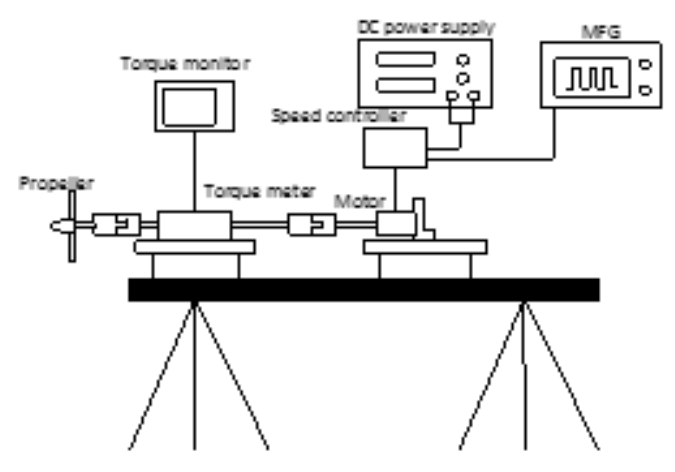

(b)

Figure 1: Experiment set up for Thrust (a) and Torque (b)

\subsection{Test of A COT Propeller}

An APC 9 x 6" commercial of the shelf propeller was tested as described in experiment test setup section of this paper. The measured thrust was corrected by adjusting drag to account for the propeller mount fixtures. The correction method applied was developed at UIUC [11] and it resulted in a slightly higher value for the measured thrust. Figure 2 shows comparison of experiment data obtained from Kyushu Institute of technology with similar data from University of Illinois Urbana Champaign. The results are in good agreement and all point lie within the $95 \%$ confidence interval.

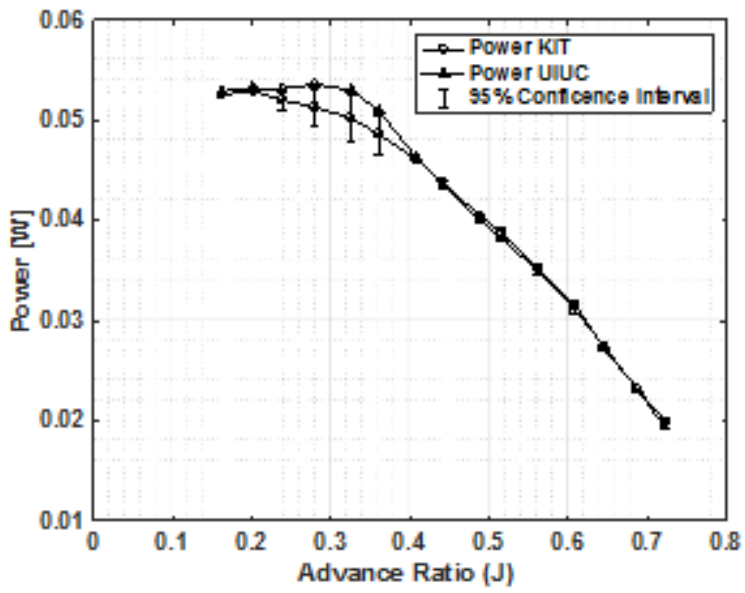

(a)

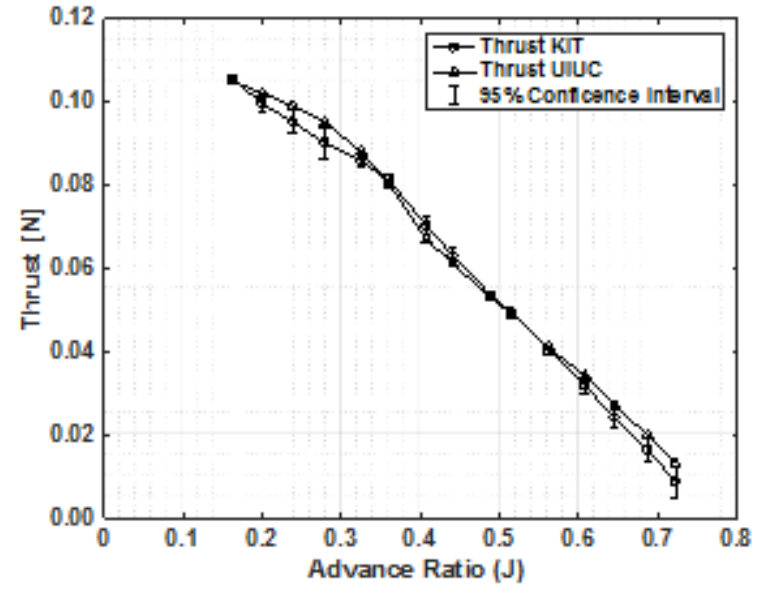

(b)

Figure 2: Power-(a) and thrus-(b) vs advance ratio experiment data from KIT and UIUC including 95\% confidence interval

\section{Propeller Design}

\subsection{Xrotor}

Xrotor iterates blade chord and twist on the condition of Minimum Induced Losses using either 2D blade momentum theory or Goldstein formulation. In both methods, lifting line is used at 
various radial stations to represent the blade geometry. Graded blade element momentum theory uses the Betz-Prandtl method for the estimation of induced velocities at the propeller plane, while the Goldstein method calculates the induced velocities by prescribing a helicoidal wake from the trailing edge vortices of the blade where Helmholtz's condition is imposed. Propeller section thrust and torque are integrated along the entire blade using equations 1 and 2 . The blade shape can only be iterated in design mode in Xrotor after which the off-design performance of the iterated blade shape is evaluated in operate mode.

$$
\begin{aligned}
& T=0.5 B \rho \int_{r_{h u b}}^{r_{t i p}} V_{R}^{2} c_{L}\left(\cos \phi-\frac{c_{D}}{c_{L}} \sin \phi\right) c d r \\
& Q=0.5 B \rho \int_{r_{h u b}}^{r_{t i p}} V_{R}^{2} c_{L}\left(\sin \phi+\frac{c_{D}}{c_{L}} \cos \phi\right) c r d r \\
& \cos \phi=\frac{\omega r-v_{t}}{\sqrt[2]{\left(\omega r-v_{t}\right)^{2}+\left(V+v_{a}\right)^{2}}} \\
& \sin \phi=\frac{V+v_{a}}{\sqrt[2]{\left(\omega r-v_{t}\right)^{2}+\left(V+v_{a}\right)^{2}}} \\
& C_{D}=\mid C_{D(0)}+d\left(C_{D}\right) / d\left(C_{L}^{2}\right) \\
& C_{T}=\frac{T}{\rho n^{2} D^{4}}\left(C_{L(0)}-C_{L}\right)^{2} \mid *\left(R e / R e_{r e f}\right)^{f}
\end{aligned}
$$

\subsection{Airfoil Considerations}

High frictional forces and low inertia associated with Low Reynolds number flows often results in adverse pressure during flow transition at the upper surface of the airfoil. The sensitivity of lowReynolds number flows makes it onerous to acquire the aerodynamic forces that govern the flow, thus, reducing the quality of $2 \mathrm{D}$ airfoil data collected by experiments. The limited understanding of the flow physics of low Reynolds number flows further extends to lower the accuracy of force coefficient estimated by numerical codes. Ultimately, uncertainties from 2-D airfoil aerodynamic data are cascaded into overall propeller performance.

A single airfoil SD7037 was used for the design of the entire design of the propeller. The 2D airfoil aerodynamic data used for the design of SD1007 was taken from experiments conducted by H.A Stokely [12].

To ensure structural integrity of the propeller the thickness of the airfoil at blade root $(\mathrm{r} / \mathrm{R}=0.175)$ was increased to $35 \%$ while at other blade stations $(\mathrm{r} / \mathrm{R}=0.287$ and 0.75$)$ a $9 \%$ thickness/chord SD7037 was maintained. Further, to check inherent trailing edge structural concerns, the airfoil trailing edge was thickened to a constant value of $0.05 \mathrm{~mm}$ along the entire blade radius, 
corresponding to $<0.5 \%$ of chords. A NACA report [13] recommends a $1 \%$ trailing-edge radius for an airfoil with $10 \%$ thickness to chord.

\subsection{Blade Shape Iteration}

Iterating a blade in Xrotor requires 3 steps: provision of atmospheric condition in which the propeller is expected to operate, linearized 2D airfoil force data, and physical propeller parameters such as dimensions.

\subsubsection{Design Atmospheric Data}

The design atmospheric data of the propeller is presented in Table 1.

Table 1: Blade design atmospheric data

\begin{tabular}{|l|l|}
\hline Atmospheric Parameter & Value \\
\hline Density $\left[\mathrm{kg} / \mathrm{m}^{3}\right]$ & 1.21 \\
\hline Speed of Sound $[\mathrm{m} / \mathrm{s}]$ & 339 \\
\hline Dynamic Viscosity $[\mathrm{Kg} / \mathrm{m}-\mathrm{s}]$ & $1.78 * 10^{-05}$ \\
\hline
\end{tabular}

\subsubsection{Linearized 2D Airfoil Force Data}

Xrotor requires linearized airfoil lift curve slope, $\mathrm{d}(\mathrm{CD}) / \mathrm{d}$ (CL2), and other specific airfoil data from which the linear behavior of the airfoil is fully established. The lift curve slope inputted in to Xrotor for this purpose was derived from H. A Stokely's experiment lift coefficient points shown in Figure 3(a). The linearized lift characteristic of SD7037 that is shown in Figure 3(a) was modeled using the lift curve slope derived from experiment data. The relationship between drag and square of lift coefficient was derived the between points shown in Figure 3(b).

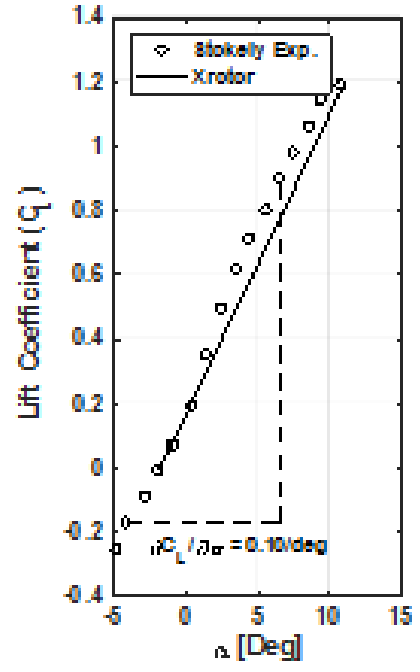

(a)

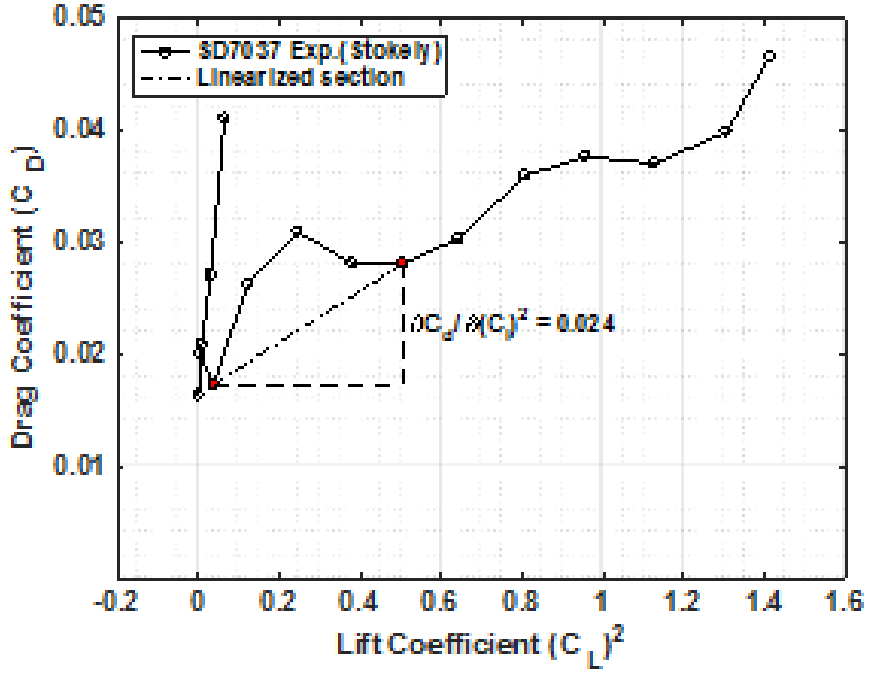

(b)

Figure 3: 2D Airfoil aero data (Stokely) showing derivation points. $\mathrm{Cl}$ vs $\mathrm{AoA}$ (a) and $\mathrm{Cd}_{\mathrm{vs} \mathrm{cl}^{\wedge} 2}$ (b) 


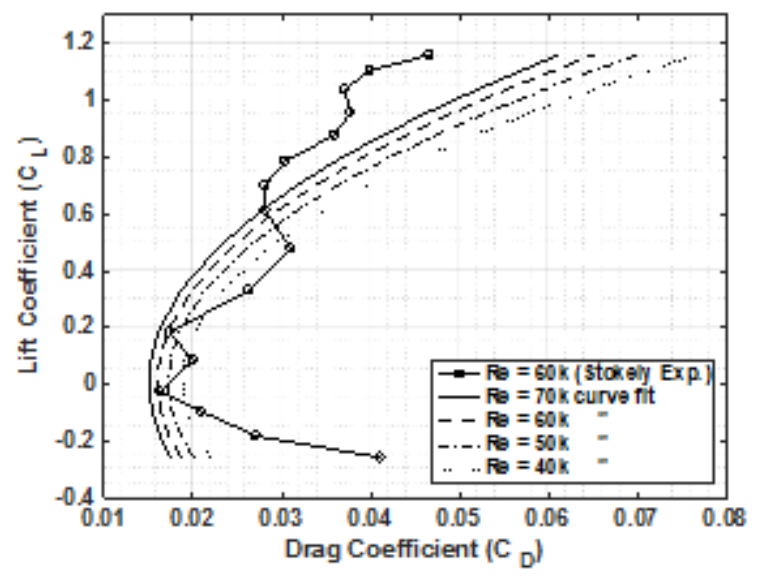

Figure 4: CL vs CD relationship with changing Reynolds number for SD7037.

Shown in Figure 3(a) is the lift curve slope from UIUC experiment and its linearized form as reconstructed in Xrotor from provided inputs. From this figure, it is clearly observed that at lift coefficient above 0.2, the linearized lift curve slope (Xrotor) slightly departs from experiment values.

Figure 4 shows CL vs CD dependence on Reynolds number obtained using equation 5. Xrotor uses equations 5 to relate CD, CL and Reynolds number to fit curve the 2D lift and drag force coefficient obtained from experiments that is also shown in Figure 4. In design mode, Xrotor accepts design inputs used in the iteration of blade shape and prediction of blade performance. Predicted performance in design mode are always in perfect agreement with corresponding performance from operate mode. The agreement between both modes is primarily because Xrotor accepts only linearized forms of 2D airfoil characteristics input.

\subsubsection{Propeller Design Input Parameters}

In design mode, the parameters found in Table 2 were used with Blade Element Momentum Theory (BEMT) implementation in Xrotor to iterate the blade pitch and chord.

Table 2: Blade design input parameters/requirement

\begin{tabular}{|l|l|}
\hline Blade parameters & Value \\
\hline Number of blades & 2 \\
\hline Tip radius [m] & 0.0875 \\
\hline Airspeed [m/s] & 8.00 \\
\hline Angular speed [RPM] & 3300 \\
\hline Constant $C_{\text {L }}$ design & 0.3 \\
\hline Lift curve slope [/deg] & 0.1 \\
\hline Solidity & 0.24 \\
\hline Chord Re @ 75\% Radius & 60,000 \\
\hline
\end{tabular}

Figure 5(a) shows the blade twist and c/R both as a function of station radius $r / R$ and Figure 5(b) shows the cumulative thrust and Reynolds number as a function of blade station radius. From Figure 5(b), it is seen that the region of the blade radius between 0.32 and 0.88 operates within 
Reynolds number range of $42 \mathrm{k}$ and $58 \mathrm{k}$ and produces about $70 \%$ of the total propeller thrust. It was desired to have a minimum variation in Reynolds number from the hub to the tip of the propeller. The blade loading of the propeller at design point is $17.43 \mathrm{~N} / \mathrm{m}^{\wedge} 2$.

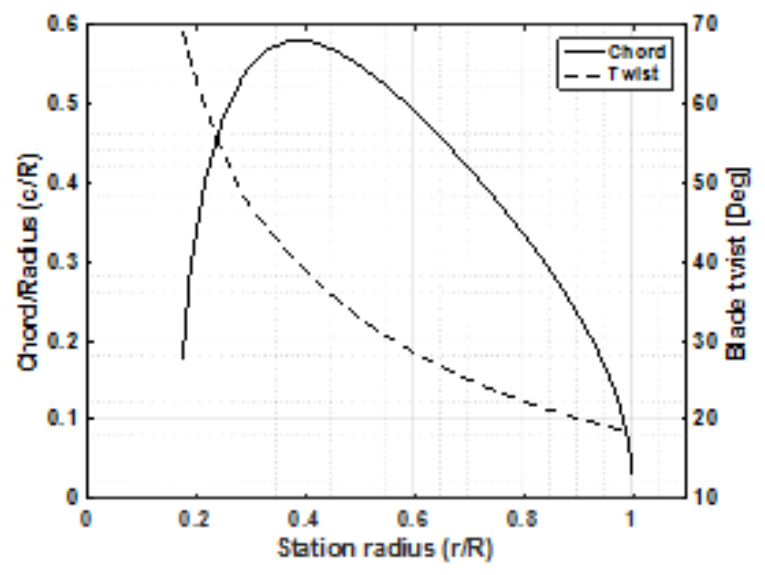

(a)

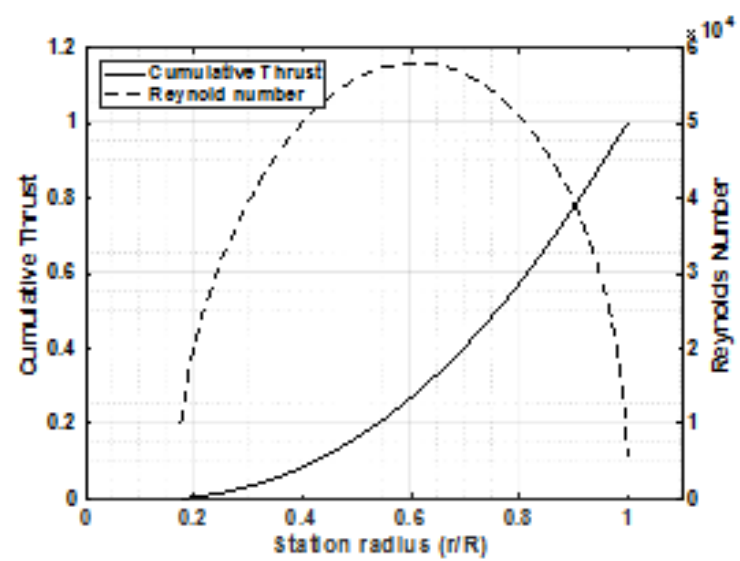

(b)

Figure 5: Iterated propeller geometry (a) and Re distribution (b)

\section{Propeller Fabrication}

The propeller was 3D printed using formlab stereolithography (SLA) printer. Formlab SLA printer is capable of delivering a resolution between $25-100$ micron in the Z-axis and 2.8 microns resolution in $\mathrm{X}-\mathrm{Y}$ axis. The printed propeller was not only smooth and required less effort to finish, but also the printer's resolution allowed for a finite trailing edge thickness of $0.05 \mathrm{~mm}$ without compromising strength. Figure 6 shows the propeller on its printing support structure after it has fully cured and hand finished. The finished propeller was designated SDL60M and it weighed 23g.
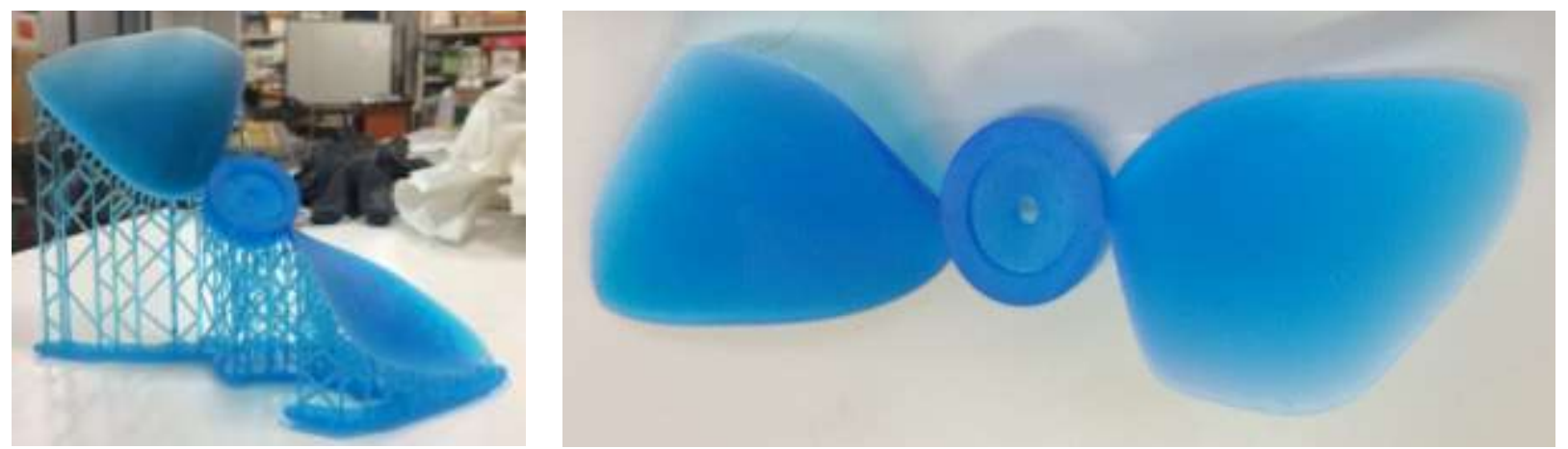

Figure 6: 3D printed propeller

\section{Test Results}

\subsection{Constant Angular Velocity Experiments and Analysis}

The wind tunnel experiment was set up as described in the experiment test set up section of this paper. SDL60M was first tested at constant angular velocity of 2300, 3300 and $4300 \mathrm{rpm}$. 


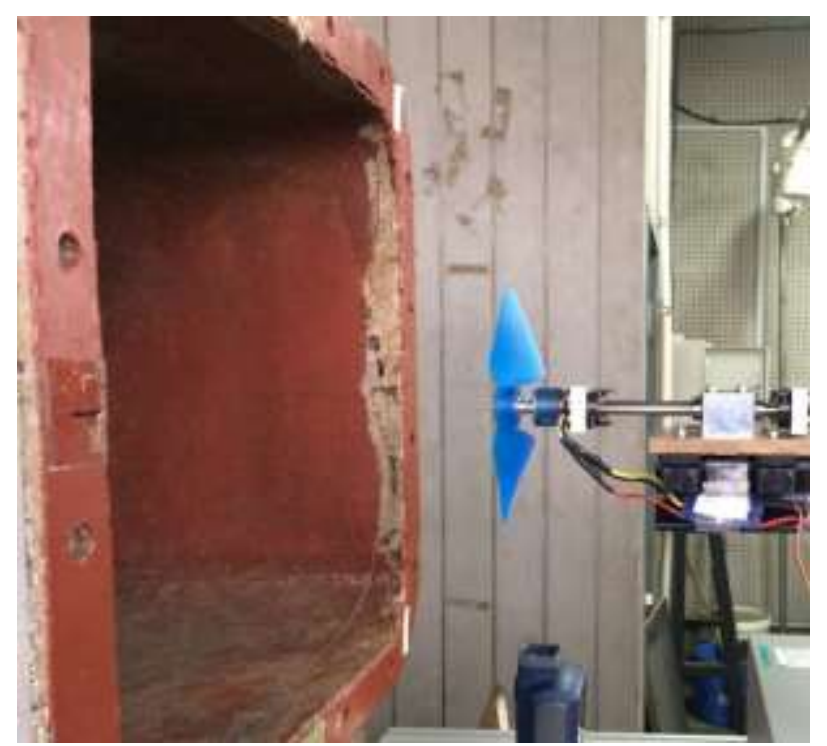

Figure 7: Propeller on Wind tunnel test stand

Figure 7 captures the experiment set up in the open test section of the wind tunnel. For each of the RPM tested, 2300, 3300, and 4300, the advance ratio swept range was from 0.3 to 1.3. The constant angular velocity test method allowed a greater range of RPM sweep, thus, the propeller performance was assessed from a lower and much wider advance ratio range.

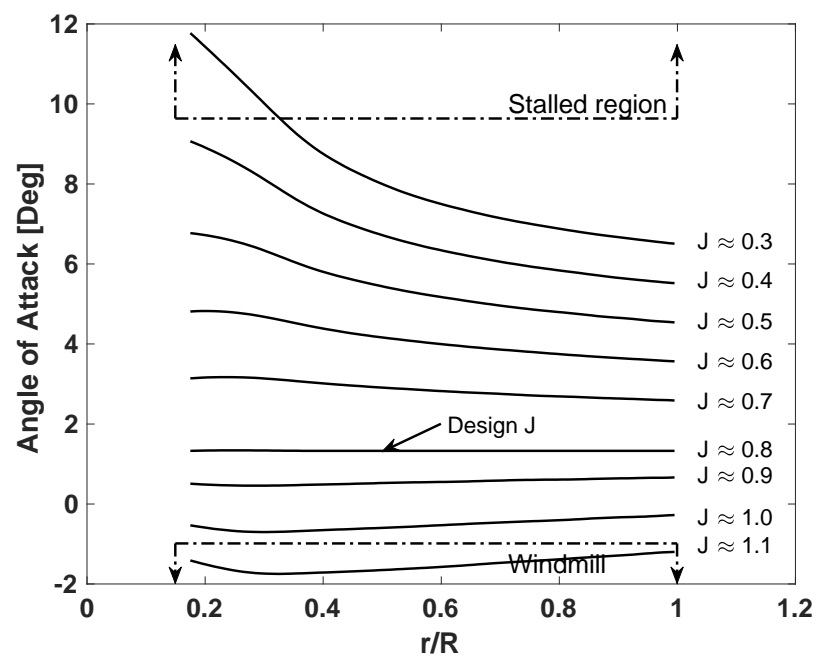

Figure 8: AoA vs station radius at constant angular velocity of 3300RPM.

The propeller was tested through the advance ratio range shown in Figure 8. At each of the advance ratios, Xrotor was used to estimate the angle of attack distribution along the blade radius. This was performed for the constant 3300RPM test case only. The radial angle of attack distribution at design point is a constant 1.3degrees. However, as advance ratio either increases or decreases, the distribution of angle of attack also increases or decreases. 


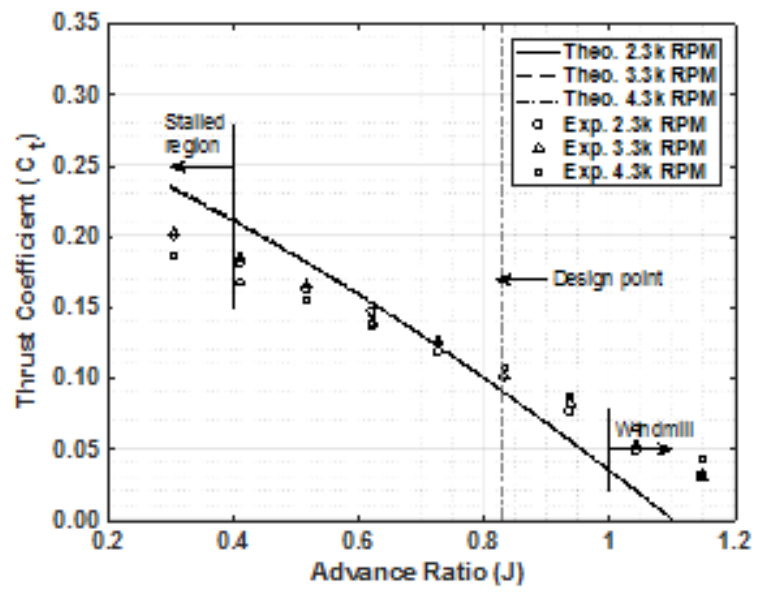

(a)

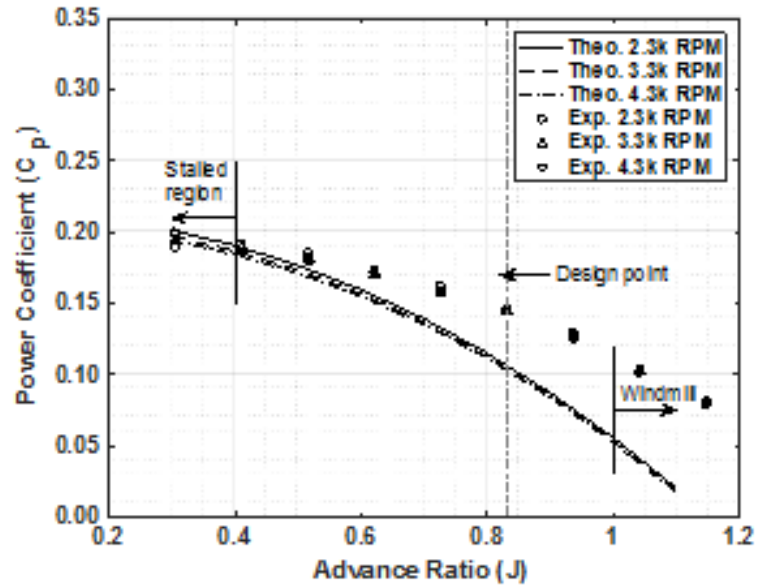

(b)

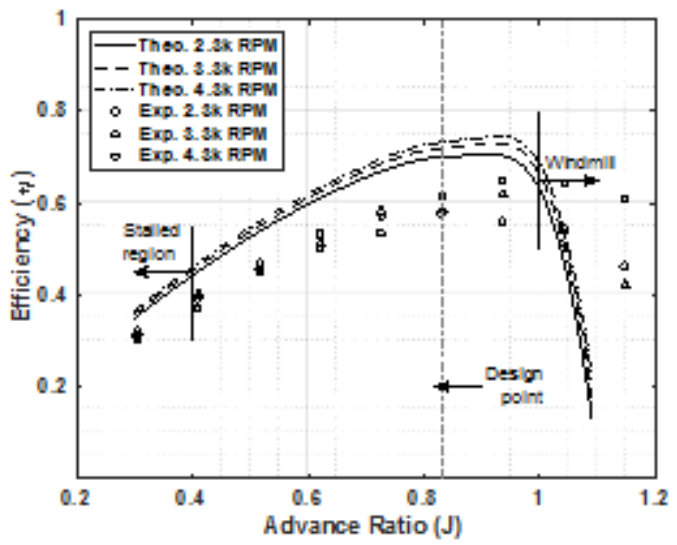

(c)

Figure 9: Constant angular velocity plots: CT (a), CP (b), and Efficiency(c)

The thrust coefficient experiment data shown in Figure 9(a) is in good agreement with theory at advance ratios near the design point but begins to depart at lower advance ratio as propeller approaches stall, which occurs around $J=0.45$. However, from Figure 9(b) significant power divergence between experiment and theory is observed. As expected, the power divergence resulted in low blade efficiency than designed as seen in Figure 9(c). At design point, BEMT efficiency prediction is $70 \%$ while $58 \%$ efficiency was obtained from experiment. An overall maximum efficiency of $65 \%$ was recorded from SDL60M wind tunnel tests carried out at constant 4300RPM shown in Figure 9(c).

\subsection{Constant Airflow Velocity Experiment}

Constant angular velocity propeller wind tunnel tests were followed by a constant airflow velocity test. 


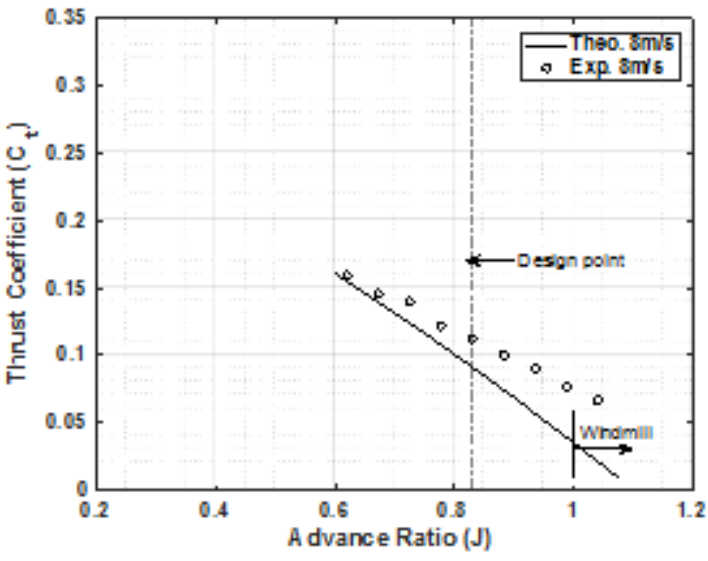

(a)

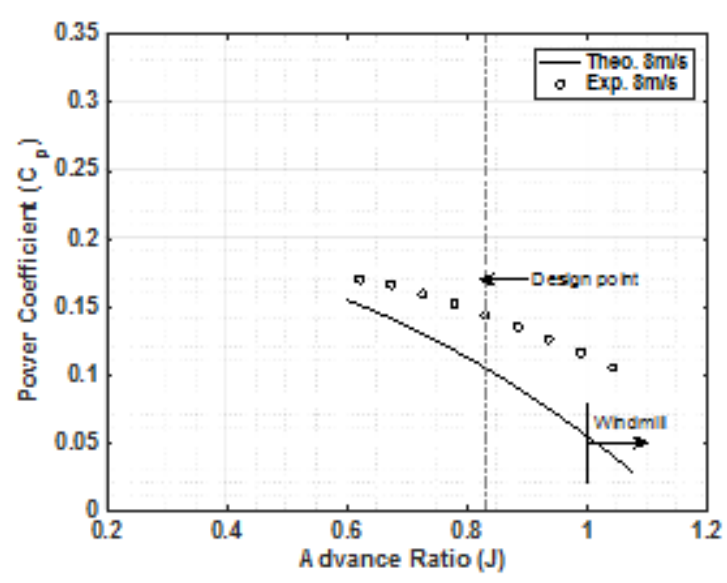

(b)

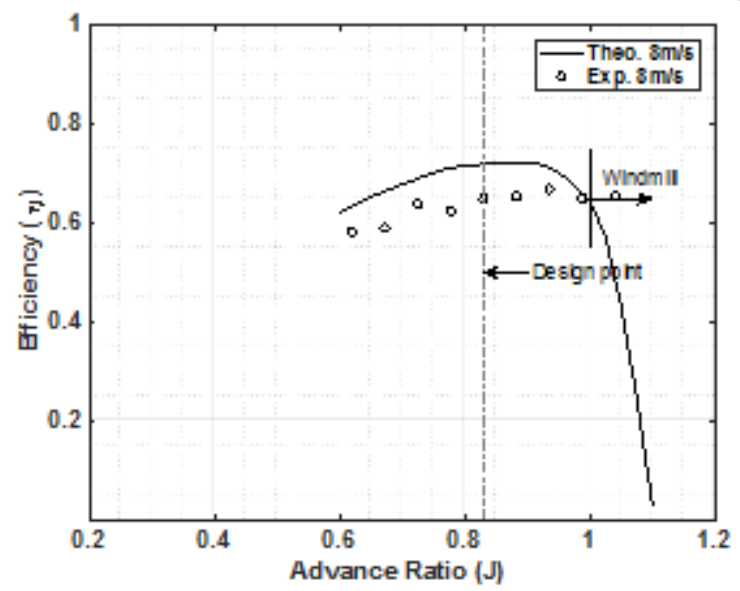

(c)

Figure 10: Constant 8m/s constant Velocity plots: CT (a), CP (b), and Efficiency(c)

This additional wind tunnel test was performed by maintaining a constant airflow velocity of $8 \mathrm{~m} / \mathrm{s}$ over the propeller while varying the angular velocity of the propeller to achieve the range of advance ratio sweep shown in Figure 10. The experiment value of CT in Figure 10(a) is about 30\% higher than theory prediction of CT at design point. Further, the power coefficient recorded from experiment shown in Figure 10(b) is 1.4 times higher than corresponding BEMT predictions. Efficiency plot of SDL60M in Figure 10(c) shows that a peak efficiency of $67 \%$ was reached from experiment at advance ratio of 0.93 , and $65 \%$ at design advance ratio of 0.83 . BEMT efficiency prediction at design point was $70 \%$.

Although the difference between the maximum efficiency obtained from the BEMT code prediction and experiment is $5 \%$ for both test cases, the difference in power is considerable for both cases. The thrust coefficient plot in Figure 10(a) also shows significant difference between BEMT code and experiment at design point. Three (3) potential reasons for design point performance discrepancy were investigated: assumption of perpendicularity of the propeller induced velocity, effect of Reynolds number, and inaccuracy of 2D aerodynamic force coefficient used for the propeller design. 


\subsection{Effect of Inflow angle calculation Assumptions on Performance}

The inflow angle $(\phi)$ is the sum of the advance ratio divided by pi $(\phi \mathrm{J})$ plus angle introduced by the induced velocities $(\phi \mathrm{i})$. The inflow distribution angle of $\phi \mathrm{J}$ and $\phi \mathrm{I}$ for SDL60M at design advance ratio is shown in Figure 11. This figure also show the plots of $\phi \mathrm{J}+10$ to $\phi \mathrm{J}-5$ decreased in steps of 5 degrees to investigate the impact of overestimated or underestimated induced velocities on propeller performance. Beside the region below $\mathrm{r} / \mathrm{R} 0.28$, having a $\phi \mathrm{I}>5$ degrees because of thick airfoil used in this region, other regions of the blade have a $\phi i<5$ degrees. Increasing and decreasing the maximum value of $\phi \mathrm{i}$ by $200 \%$ is equal to $\phi \mathbf{J}+10$ and $\phi \mathbf{J}-10$ respectively. However, from Figure $12(\mathrm{a} \& \mathrm{~b})$, it is seen that increasing $\phi i$ by 10 degrees at all blade section radius results in a thrust coefficient that is below and power coefficient that is above experiment values. Decreasing $\phi i$ will have the opposite effect. By using high and unlikely value for $\phi \mathrm{i}$, the assumption of perpendicularity of induced velocities is eliminated as a major contributor to the huge discrepancy observed in the measured performance data of SDL60M.

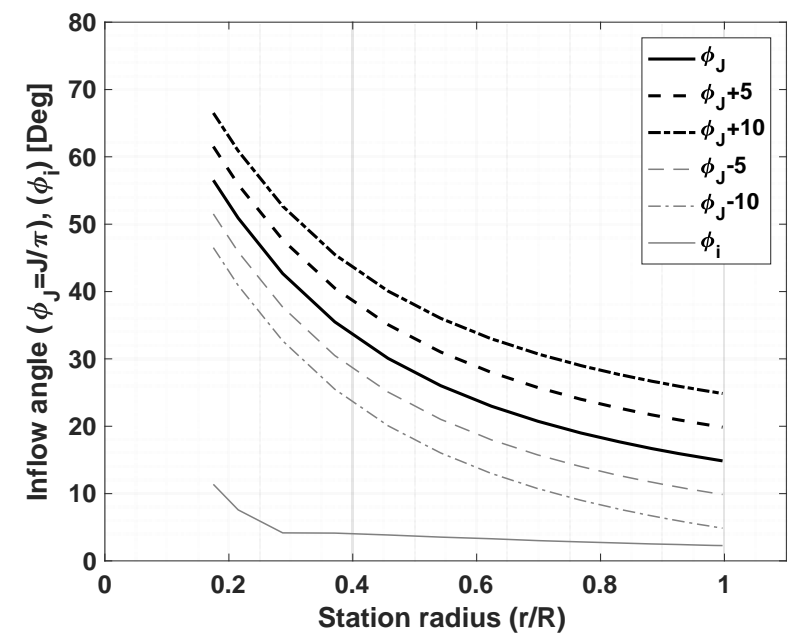

Figure 11: Inflow angle $(\phi \mathrm{J}=\mathrm{J} / \pi)$ Vs blade station radius $\mathrm{r} / \mathrm{R}$

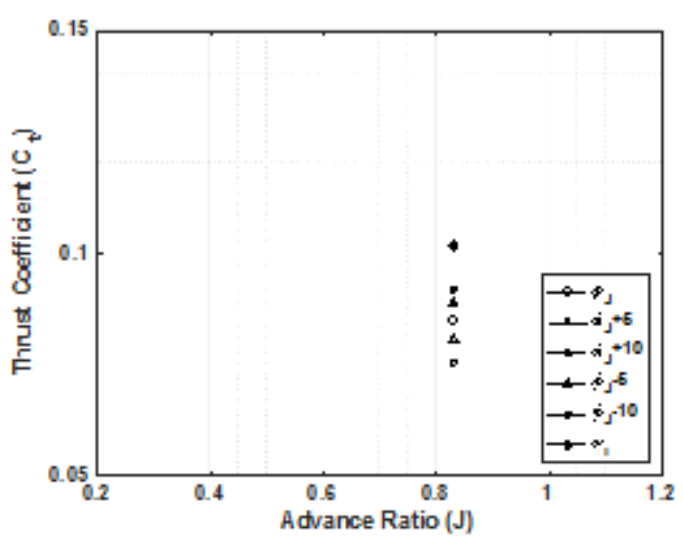

(a)

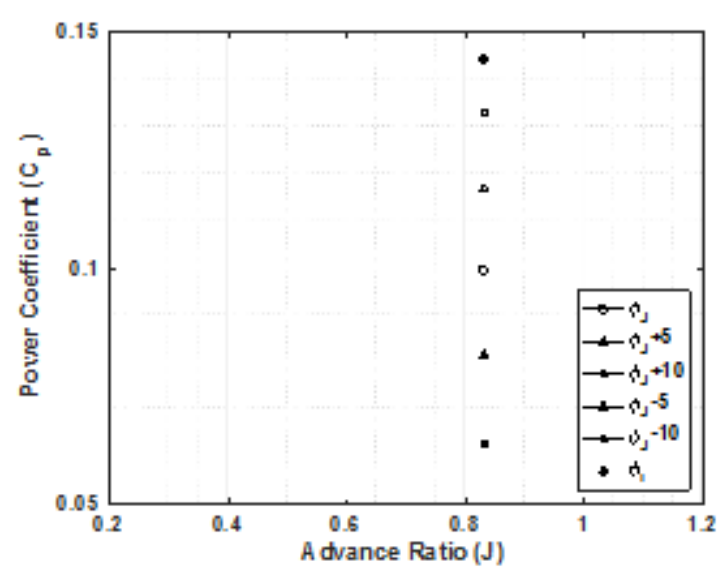

(b)

Figure 12: CT Vs J (left) CP Vs J (right) 


\subsection{Effect of Reynolds Number and Aerodynamic Force Data on Performance}

The lift curve slope used in the iteration of SDL60M in the BEMT code was derived from a wide angle of attack range shown in Figure 3(a). This range is representative of a wide propeller operation regime, however, it does not accurately represent specific local lift coefficient at corresponding angle of attacks. To investigate the effect of a narrower and more representative local lift curve slope around the design point, a new lift curve slope was derived from the nearest two points around the design lift coefficient. The resulting lift curve was found to be much higher than the lift curve slope used in the iteration of SDL60M. Figure 13(a) shows the exact points from which a more representative local lift curve slope was derived. CL points from which the lift curve slope was derived was also used in deriving $\mathrm{d}(\mathrm{CD}) / \mathrm{d}$ (CL2), which in turn was to establish the linear relationship between $C L$ and $C D$ shown in Figure 13(b). At $C L=0.3$, good agreement between $C L$ and $C D$ is observed in Figure 13(a \& b).

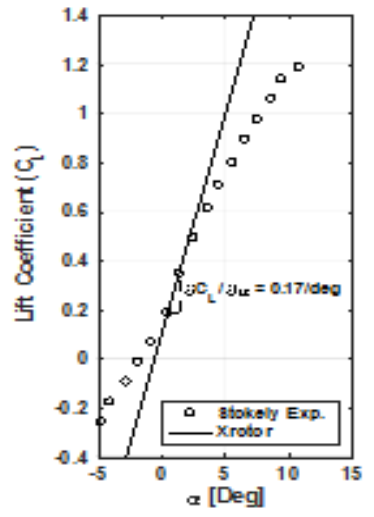

(a)

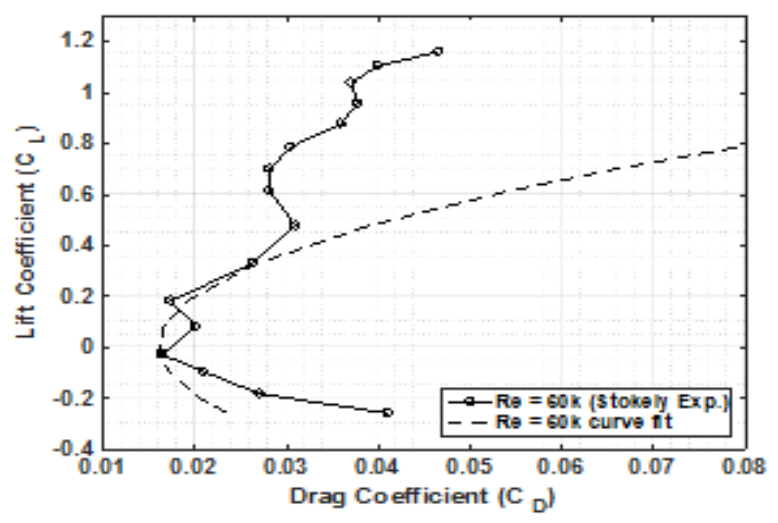

(b)

Figure 13: Recomputed lift curve slope (a) and linear relationship between CL and CD (b)

The re-computed lift curve slope in Figure 13 and d(CD)/d(CL2) were inputted to Xrotor, and used in the performance analysis of the previously iterated blade shape (SDL60M) shown in Figure 5. The blade shape performance analysis was performed in operation mode in Xrotor. Re-analyzing the shape of the previously printed SDL60M with the new linear airfoil force coefficients was chosen as an alternative to fabricating an entirely new propeller design.

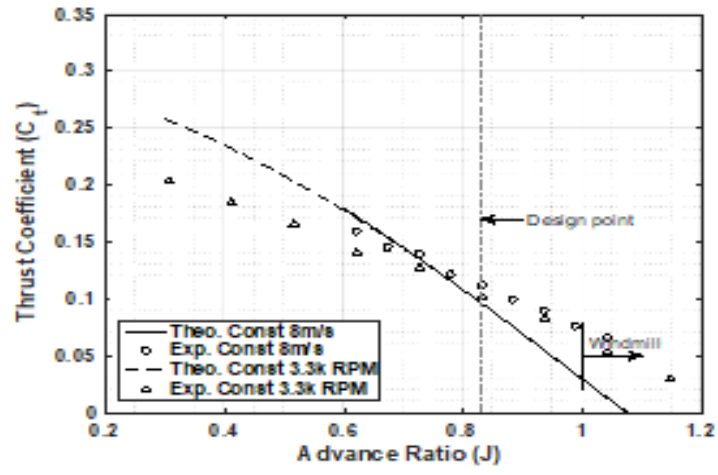

(a)

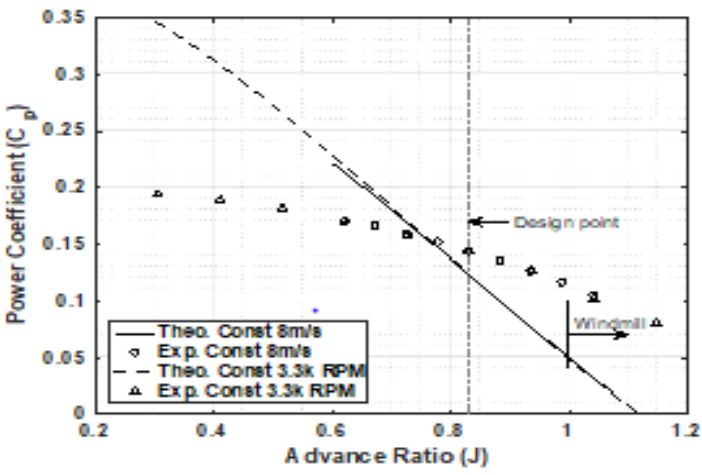

(b)

Figure 14: Ct vs J (a) and Cp vs J (b) for $\delta C L / \delta \alpha=0.17$ 
Figure 14 shows the $\mathrm{CT}$ and $\mathrm{CP}$ plots of the blade analyzed at constant linear velocity and constant angular velocity. For the purpose of comparison, the experiment CT and CP shown in Figure 14 were re-presented from Figure 9 and Figure 10. From Figure 14, it is observed that BEMT predictions show a significantly improved CT and CP agreement at design point. However, at advance ratios other than design, theory $\mathrm{CT}$ and $\mathrm{CP}$ begin to depart from experiment.

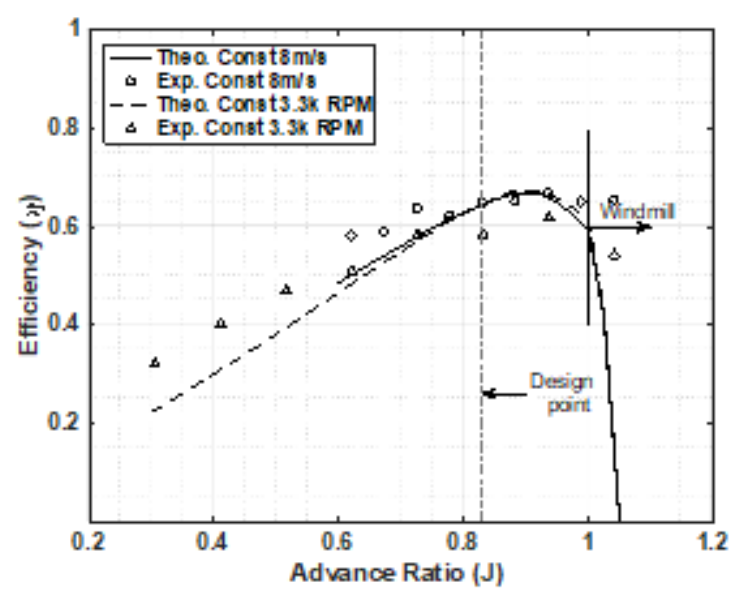

(a)

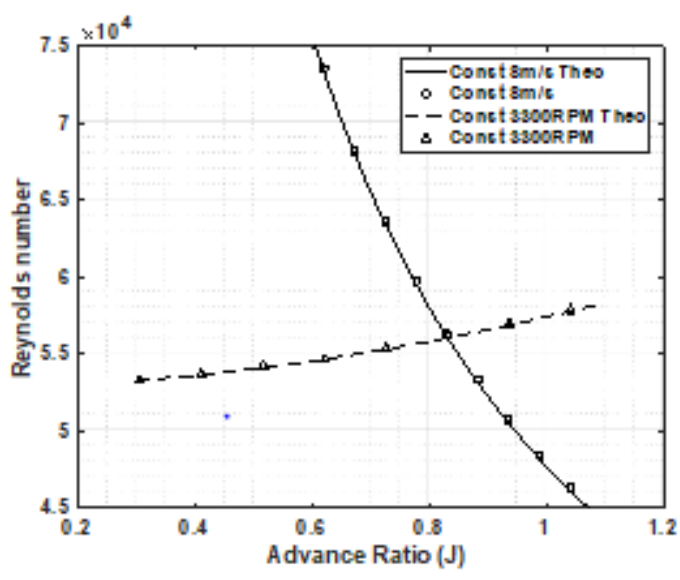

(b)

Figure 15: $\eta$ vs J (a) and Reynolds number vs J (b) for $\delta C L / \delta \alpha=0.17$

\subsubsection{Reynolds Number Effects}

Figure 15(a) shows efficiency as a function of advance ratio, and the Reynolds number profile of the constant airflow velocity and constant angular velocity experiments in Figure 15(b). Reynolds number analysis is explored in two ways: The effect of variation between the theoretical and experimental values of propeller Reynolds number found in Figure 15(b) on efficiency in Figure 15(a) and the effect of Reynolds number as a result of two different experiment methods dependence of Reynolds number on blade performance.

As observed in Figure 15(b), there is good agreement between theory and experiment reynolds number values of SDL60M. However, in Figure 15 (a), plots of efficiency shows discrepancy between corresponding pairs of theory and experiment data.

The efficiency plots of experiment data obtained from both test methods at same advance ratio shows slight difference due to variation in the respective operation Reynolds numbers, which was minimized from design by ensuring minimum variation from propeller hub to tip radius. The $75 \%$ station Reynolds number plots of the propeller as a function of advance ratio shows a range of about 20k between the constant airflow velocity and angular velocity operation cases at advance ratio of 0.61 . Hence, it can be inferred that the dependence of propeller performance on Reynolds number is small and not the major contributor to the performance discrepancy between theory and experiment. McCormick [14] asserts that for propellers, dependence of lift curve slope and drag/lift ratio on Reynolds number is small and can be considered negligible. 


\subsubsection{Aerodynamic Force Data Effects}

The region of the blade from $60 \%$ station radius outbound to the blade tip generates more than $75 \%$ of the total propeller thrust. Moving from inbound of the propeller radius to outbound regions, the local flow angle $\phi$ of the blade reduces, hence $\operatorname{Cos}(\phi)$ in equations 1 and 2 dominates $\operatorname{Sin}(\phi)$. Since CL and CD were the only changed parameters, a theoretical re-analysis and re-evaluation of the performance of SDL60M blade shape carried out under this condition. In re-analyzing the blade shape, the linearized values of CL and CD relative to corresponding experiment values applied to equation 1 and 2 could have four effects on the overall predicted thrust delivered and power absorbed by the propeller:

1) If the linearly obtained value of CL is lower than the experiment values while the linear and experiment values of $\mathrm{CD}$ agree, the estimated propeller thrust and power would be lower than experiment values.

2) If the linearly obtained value of CL is higher than the experiment values while the linear and experiment values of CD agree, the estimated thrust and power would be higher than experiment values.

3) If a lower than experiment value of CD is used in the re-analysis of the blade while the linear and experiment values of CL agree, the estimated thrust would be higher and power lower than experiment.

4) Lastly, if a higher than experiment value of CD is used to re-analyze a blade shape while the linear and experiment values of CL agree, the estimated thrust would be lower and power higher than experiment.

The analysis above assumes that 2D airfoil experiment data are accurate. SDL60M was iterated using a lift curve slope of $0.10 / \mathrm{deg}$ and from Figure 8, the design CL of 0.3 corresponds to angle of attack of $1.3 \mathrm{deg}$.

Table 3: SD7037 airfoil experiment data \& its linearized form $(\mathrm{a} 0=0.10 / \mathrm{deg}) @ \mathrm{Re}=60 \mathrm{k}$

\begin{tabular}{|l|l|l|l|l|}
\hline & $\mathrm{C}_{\mathrm{L}(@ \mathrm{a} 0=0.10)}$ & $\mathrm{C}_{\mathrm{L}(\mathrm{E})}$ & $\mathrm{C}_{\mathrm{D}(@ \mathrm{CL}=0.3)}$ & $\mathrm{C}_{\mathrm{D}(\mathrm{E})}$ \\
\hline @ AoA =1.3deg & 0.30 & 0.34 & 0.020 & 0.026 \\
\hline
\end{tabular}

Table 3 captures the linearized and experiment values of lift and drag coefficient of SD7037 at angle of attack of 1.3deg, taken from Figure 3(left) and Figure 4(left). From Table 3, it is seen that while the linearized lift coefficient, $\mathrm{CL}(@ \mathrm{a} 0=0.10)$, used in the iteration of the blade is equal to 0.3 , the corresponding experiment value, $\mathrm{CL}(\mathrm{E})$, is $11 \%$ higher. Consequently, where the linearized CL is 0.3 , the corresponding linear $\mathrm{CD}(@ \mathrm{CL}=0.3)$ is 0.02 , which is $20 \%$ less than experiment value of $\mathrm{CD}(\mathrm{E})$ compared at same $\mathrm{CL}$. The combined effect of lower linearized forms of aerodynamic data used for the iteration of SDL60M resulted in lower-than-experiment values of CT and CP seen in Figure 9(b) and Figure 10(b) at design point.

Table 4: SD7037 airfoil experiment data $\&$ its linearized form $(\mathrm{a} 0=0.17 / \mathrm{deg}) @ \mathrm{Re}=60 \mathrm{k}$

\begin{tabular}{|l|c|c|c|c|}
\hline & $\mathrm{C}_{\mathrm{L}(@ \mathrm{a} 0=0.17)}$ & $\mathrm{C}_{\mathrm{L}(\mathrm{E})}$ & $\mathrm{C}_{\mathrm{D}(@ \mathrm{CL}=0.5)}$ & $\mathrm{C}_{\mathrm{D}(\mathrm{E})}$ \\
\hline @ $\mathrm{AoA}=1.3 \mathrm{deg}$ & 0.30 & 0.30 & 0.025 & 0.026 \\
\hline
\end{tabular}


Again, the linearized and experiment aerodynamic data in Table 4 were extracted from Figure 13 and Figure 4. In this case, the experiment and re-computed linear estimates for CL and CD are in excellent agreement as shown in Table 4. Despite the agreement between experiment and linear estimates of the force coefficients, discrepancies in performance especially in power are observed in Figure 14(a) and Figure 14(b).

Thompson [15], Michaelis [15], and Bregman [16] all performed experiments on SD7037 at Reynolds number of 60k. The results of their experiments are shown in Figure 16. From this figure, it is observed that while the lift curve slope of all experiments have similar profiles, the drag profiles show considerable variation between each other. The lift curve slopes of all the experiments at higher angle of attack $(>12 \mathrm{deg}$ not shown here) reveal the presence of Aerodynamic hysteresis, indicating the presence of laminar separation bubble. Selig [17] suggests that laminar bubble is responsible for the drastic increase in airfoil drag coefficient mostly occurring about mid lift region. For propellers that operate in low Reynolds flight regime, it is almost certain that within its operational envelope some region of the propeller would contain laminar separation bubble. The presence of laminar separation bubble, which is observed around mid-lift region of Figure 16(b), may act to degrade the overall propeller performance.

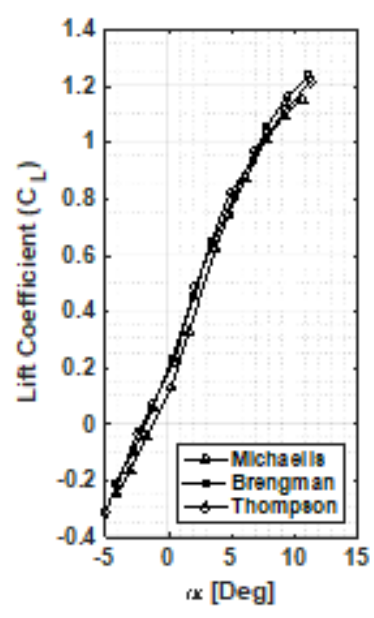

(a)

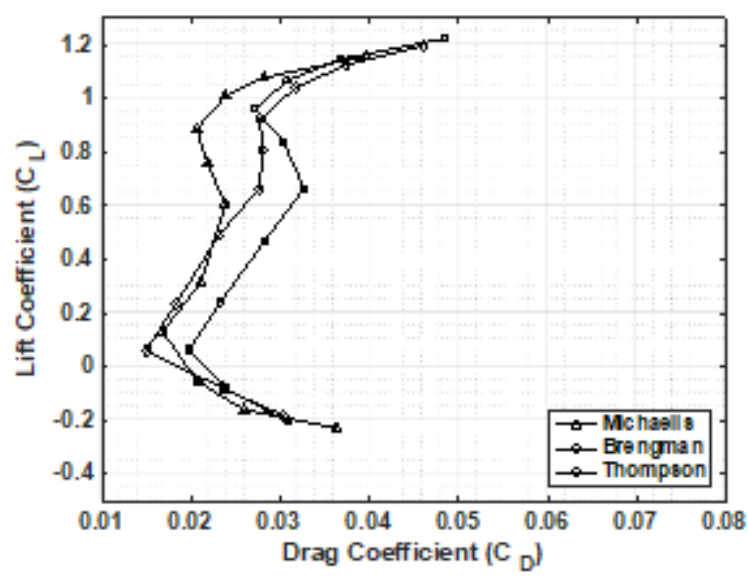

(b)

Figure 16: CL Vs $\alpha$ (a) and CL Vs CD (b) for SD7037 at Re=60k

The local CL and CD of the blade shown in Table 4 at design point are equal to their respective linearized values. Given the sensitivity of overall propeller performance to CL and CD, it can be concluded that accurate 2D force coefficients are necessary for accurate prediction of propeller thrust and power. Figure 16 (a \& b) clearly indicates that inaccuracy in the measured airfoil aerodynamic forces are considerable especially in drag. Taken together, the 2D airfoil aerodynamic measurement inaccuracies, which is due to non-linearity associated with low Reynolds number flows, is a major contributor to the propeller performance discrepancy recorded at these low Reynolds numbers.

\section{Conclusion}

Blade element momentum theory was used in the design of a 2-bladed propeller with solidity of 0.24 and chord based Reynolds number of $\approx 60 \mathrm{k}$ calculated at $75 \%$ radius and advance ratio of 
0.83. A single 2D experiment data of SD7037 at 60k Reynolds number was employed in the design of more than $75 \%$ of the entire blade, which was based on minimum propeller induced losses. From blade design, it was ensured that above $50 \%$ of the entire blade radius operated between 40k - 60k Reynolds numbers at design advance ratio. A design goal of the propeller was to minimize variation in Reynolds number from hub to tip radius. Propeller tests were conducted at Kyushu Institute of Technology wind tunnel facility in two ways: A constant angular velocity was maintained while airflow velocities over the propeller was changed at each data point; and a constant airflow over the propeller while changing angular velocity of the propeller at required data points. Performance discrepancy between blade element momentum theory predictions and data from wind tunnel experiment was observed.

The constant angular velocity and air velocity wind tunnel tests both achieved a maximum efficiency of $65 \%$, and respective efficiency of $58 \%$ and $65 \%$ at design point. However, blade element momentum theory predictions at design point were about $70 \%$ efficiency for the both cases. While the maximum efficiencies reached do not significantly differ from theory predictions, thrust and power experiment data show considerable discrepancy with theory at design point.

Because Xrotor accepts airfoil aerodynamic input in linearized forms only, the effect of drag and lift coefficients on propeller performance was investigated in detail by re-computing the lift curve slope from a narrower range that gave a more representative value for the local design angle of attack. Performance of an earlier iterated blade shape, which was fabricated and tested, was repredicted in operation mode in Xrotor by replacing the lift and drag force coefficients with the recomputed value. The re-computed airfoil force coefficients agreed excellently with experiment data and predicted propeller performance showed better agreement especially in thrust. However, discrepancy in power remained considerable.

For low Reynolds number operating propellers, a major source of performance discrepancy between theory and experiment is inaccurate lift and drag coefficient used in the blade shape iteration. Investigation showed that blade performance is especially sensitive to lift and drag coefficient, and an unrepresentative value contributes to discrepancies between theory predictions and experiment data. Non-linearity associated with airfoil aerodynamic data that are not captured by linearization result in a less representative modeling of the airfoil force coefficient and consequently, discrepancy in propeller performance between theory and experiment.

\section{Declaration of Interests}

None

\section{Acknowledgement}

This work was done in the collaborative research 17UKSG-007330 with Japan Aerospace and Exploration Agency.

\section{References}

[1] Betz, Albert. The Theory of the Screw propeller. s.l.: National Advisory Committee for Aeronautics, 1921. 
[2] On the Vortex Theory of Screw Propellers. Goldstein, Sydney. s.1.: Royal Society Publishind, 1929.

[3] Drela, Mark. qprop_theory.pdf. [Online] 06 2006. [Cited: 03 10, 2016.] http://web.mit.edu/drela/Public/web/qprop/qprop_theory.pdf.

[4] Drela, Mark and Youngren, Harold. Xrotor_doc.txt. [Online] 11 13, 3003. [Cited: 02 28, 2016.] web.mit.edu/drela/Public/web/xrotor/xrotor_doc.txt.

[5] Muller, Thomas J. Low Reynolds Number Vehicles. University of Notre Dame. Indiana: Advisory Group For Aerospace Research And Development, 1985.

[6] Test, Analysis and Design of Propeller propulsion Systems for MAVs. Youngren, Harold. Florida: AIAA, 2011. AIAA 2011-876.

[7] Design, Fabrication, Analysis and Testing of a Micro Vehicle Propeller. Smedresman, Adam, Yeo, Derrick and Shyy, Wei. Hawaii: AIAA, 2011. AIAA 2011-3817.

[8] Reynolds Number Effects on the performance of Small-scale Propellers. Deters, Robert, Ananda, Gavin and Selig, Michael. Georgia: AIAA, 2014. 2014-2151.

[9] Development of the Black Widow Micro Air vehicle. Grasmeyer, Joel and Keennon, Mathew. Nevada: AIAA, 2001. 2001-0127.

[10] The Design of Airfoils at Low Reynolds Numbers. Michael, Selig. Nevada: AiAA, 1985. 85-0074.

[11] Michael, Selig and Ananda, Gavin. UIUC Propeller Database - Volume 1. University of Illinois Urbana Champaign. [Online] 01 11, 2015. [Cited: 06 22, 2017.] http://mselig.ae.illinois.edu/props/volume-1/propDB-volume-1.html.

[12] Christopher, Lyon A, et al. Summary of Low-Speed Airfoil Data. Virginia: SoarTech Publication, 1997. Vol. 3.

[13] Joseph, Herrig, Emery James and Erwin John. Effct of Section Thickness and Trailing-edge Radius on the Performance of NACA 65-series Compressor Blades in Cascade at Low Speeds. Washington: National Advisory Committee for Aeronautics, 1951.

[14] McCormick, Barnes. Aerodynamics of V/STOL Flight. London: Academic Press INC., 1967.

[15] Michael, Selig, et al. Summary of Low-Speed Airfoil Data. Virginia: SoarTech, 1995. Vol. 1.

[16] Michael, Selig, et al. Summary of Low-Speed Airfoil Data. Virginia: SoarTech Publications, 1996. Vol. 2.

[17] Michael, Selig, Donovan and Fraser. Airfoils at Low Speeds. Virginia: H A Stokely, 1989.

\footnotetext{
*Corresponding author.

E-mail address: p595109j@ mail.kyutech.jp
} 